\title{
Survey on Measurement Methods for IPv6 Deployment
}

\author{
V. Iró ${ }^{1, *}$, G. Lencse ${ }^{1}$ \\ ${ }^{1}$ Budapest University of Technology and Economics, Department of \\ Networked Systems and Services \\ Magyar tudósok körútja 2., 1117 Budapest, Hungary
}

*E-mail: iroviktoria@gmail.com

Abstract: $\quad$ Nowadays we use Internet Protocol (IP) versions 4 (IPv4) and 6 (IPv6) at the same time. It is very difficult to determine the rate of IPv6 and the state of its deployment exactly. In our survey, paper we present some tools and methods that can be used to estimate the proportion of IPv6. First, we will show some server side methods that mostly use provider-measurable data, so they may not be available to everyone. Next, we introduce various client-side tools that can be used in a much broader context, and then present the use of survey methods. Then we are reviewing some researches, which provide more comprehensive insight into the transition to IPv6 using complex measurement methods. Finally, we compare and categorize the presented metrics based on the availability of data sources and the extent of the measurement duration. We know that there is clearly no best among the various methods. Choosing the right technique depends greatly on the data and tools available to the researcher.

Keywords: IPv6, IPv6 transition, IPv6 readiness, measurement techniques, survey

\section{Introduction}

IANA (Internet Assigned Numbers Authority) distributed the last unallocated IPv4 blocks to Regional Internet Registries (RIR) February 3, 2011 [1], and thus the IPv4 address space was officially depleted. In practice, only addresses from previously allocated blocks can change hands [2] at relatively high prices. However, the really big technological explosions are just coming. We will soon be connecting 
almost all of our devices to the World Wide Web (Internet of Things), but not all computers currently have their own public IPv4 addresses. This problem was expected in the '90s, so the first usable version of IPv6 was completed in 1998 (RFC $2460[3])$.

Against the expectations, there has been no full transition to IPv6 over the last 20 years [4]. The progress of IPv6 deployment has been studied by several research teams at different times. In this paper, we survey the available measurement methods and metrics based on some of these studies.

\section{Server Side Measurement Methods}

Grégr, Švéda and Podermanski [5] presented several measurement methods. One of them is based on the number of IPv6 prefixes reserved for autonomous systems (AS-es). As Karpilovsky et al. [6] have already shown in an earlier study, most of the allocated prefixes are not actually used yet, but reserved for later use. So, having an IPv6 prefix allocated does not mean that IPv6 is actually available to the clients of that ISP. Although we cannot accurately determine the number of IPv6 addresses actually in use, it is still good to estimate the rate of increase in the deployment to IPv6.

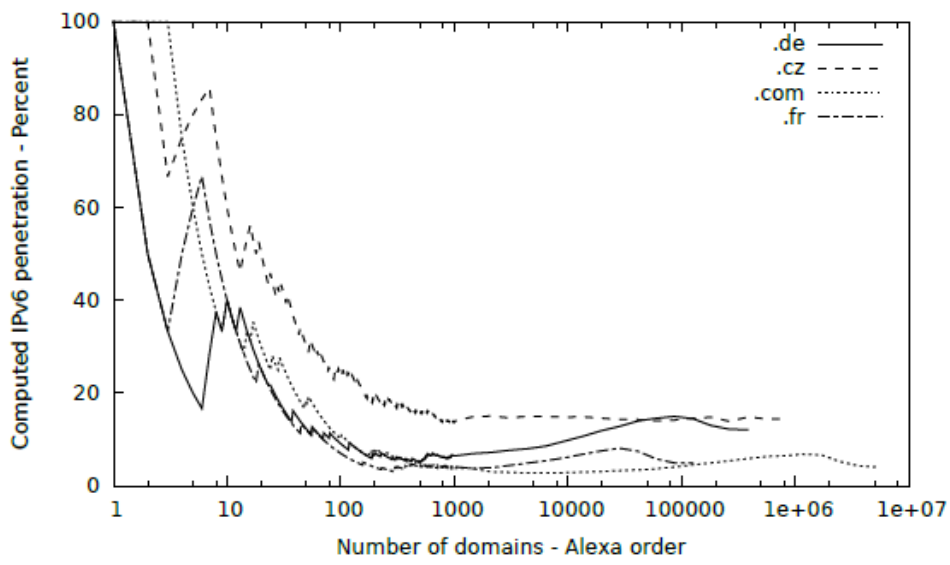

Figure 1. Dependence of the IPv6 ratio on the number of domains [5]

Grégr et al. [5] also examined the DNS Resource Record (RR) field of the packets during their measurements, if it was of type "A" (IPv4) or "AAAA" (IPv6). They also measured the time it took for the server to respond to the first SYN message, when the connection was established. We can see that IPv4 had better response times at first, but later it changed to the opposite. Of course, the measurements took the 
different distances into account, as well as the asymmetry of the routing, the different hop numbers and other distortion factors.

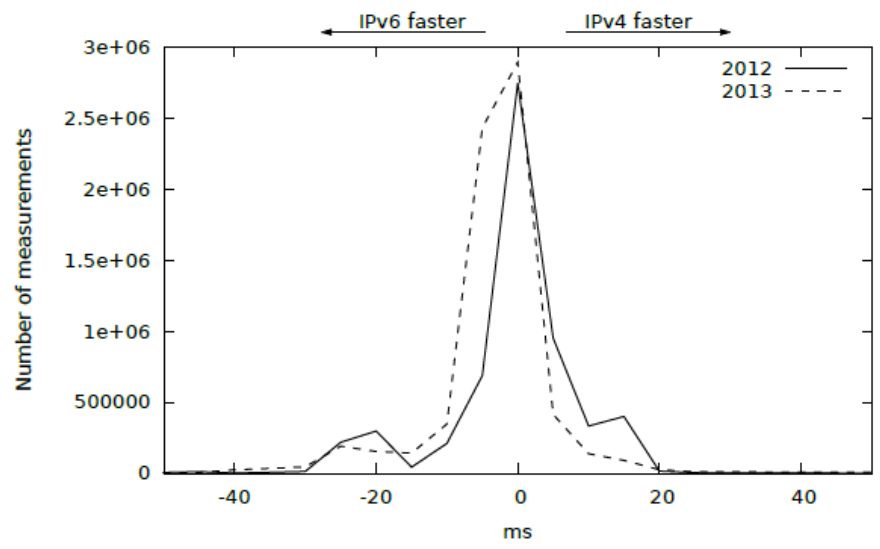

Figure 2. IPv4, IPv6 performance - number of measurements [5]

Another server-side measurement tool is Amazon's Alexa Rankings webpage [7], which containes domain names for the most popular websites. The list used to include the first one million most popular domain names, but recently we can only see the top 500 in the list for free, and we have to pay for the rest. A solution to this problem can be the list available from [8].

\section{Client Side Measurement Methods}

Geoff Huston [9] investigated the number of IPv6 clients using Google Analytics [10]. Complementing an existing Google Analytics code, he ran three simple tests in the client browser when loading the distinguished website. The first of the background tests loaded a 1 pixel white gif if the DNS name of the image could only be resolved to an IPv6 address, so there was only "AAAA" Resource Record for that DNS name (Table 1: The client can/cannot retrieve an IPv6-only URL). The second test was also responsible for loading a 1 pixel white gif if the image was available via IPv4 and IPv6 (Table 1: The client can/cannot retrieve a dual stack URL). The third test showed an image similar to the previous one if it detected only an IPv4capable device (Table 1: The client can/cannot retrieve an IPv4-only URL). The Analytics server analyzed the results of each test individually (successful or not) based on reports returned by clients and then automatically generated the appropriate statistics by event (Table 1). 
Table 1. IPv6 Measurement Tracked Event Totals [9]

\begin{tabular}{|c|c|c|}
\hline Event & Explanation & Count \\
\hline IPv4: yes & \multirow{2}{*}{ The client can/cannot retrieve an IPv4-only URL } & 2034 \\
\cline { 1 - 1 } IPv4: no & & 105 \\
\hline Dual: yes & \multirow{2}{*}{ The client can/cannot retrieve a dual stack URL } & 2013 \\
\cline { 1 - 1 } & & 108 \\
\hline Dual: no & \multirow{2}{*}{ The client can/cannot retrieve an IPv6-only URL } & 209 \\
\cline { 1 - 1 } & & 1914 \\
\hline IPv6: no & &
\end{tabular}

The advantage of this method is that it is simple and does not require changes to the content.

Huston [11] used an ad and the associated Flash code to crawl IPv6-capable clients. The ad that appears in the user's browser generates a unique DNS name that is returned to the server in a pair of URLs. The uniqueness of the strings ensures that each DNS query is a completely new DNS query, which cannot be answered from some cache. A brief summary of the measurement results is given in the Table 2, detailed [11].

Table 2. Counting IPv6 in the DNS - Short Results [11]

\begin{tabular}{|l|c|}
\hline \multicolumn{1}{|c|}{ Question } & Result \\
\hline $\begin{array}{l}\text { How many DNS resolvers generated queries in this } \\
\text { experiment over IPv4? }\end{array}$ & 111538 \\
\hline $\begin{array}{l}\text { How many DNS resolvers also generated queries in this } \\
\text { experiment over IPv6? }\end{array}$ & 5225 \\
\hline How many client experiments completed IPv4 DNS queries? & 2300384 \\
\hline How many client experiments completed IPv6 DNS queries? & $432632(19 \%)$ \\
\hline $\begin{array}{l}\text { How many unique IP addresses completed web fetches for } \\
\text { objects named in the experiment? }\end{array}$ & 890920 \\
\hline $\begin{array}{l}\text { How many clients were able to perform web fetches that } \\
\text { required IPv6 DNS resolvers? }\end{array}$ & $161125(16 \%)$ \\
\hline
\end{tabular}

Pickard, Stocks, Hamman and Robinson [12] used Nephos's V6Sonar platform [13] to monitor websites in 2015. During the measurement, they searched for web pages that are (also) accessible over IPv6. Measurements were conducted by various agents at 6 locations for 6 days: Atlanta, Seattle, Hong Kong, Netherlnads, Singapore and Slovenia. The results of the measurement are shown on Figure 3. 
V. Iró and G. Lencse - Acta Technica Jaurinensis, Vol. 13, No. 2, pp. 112-130, 2020

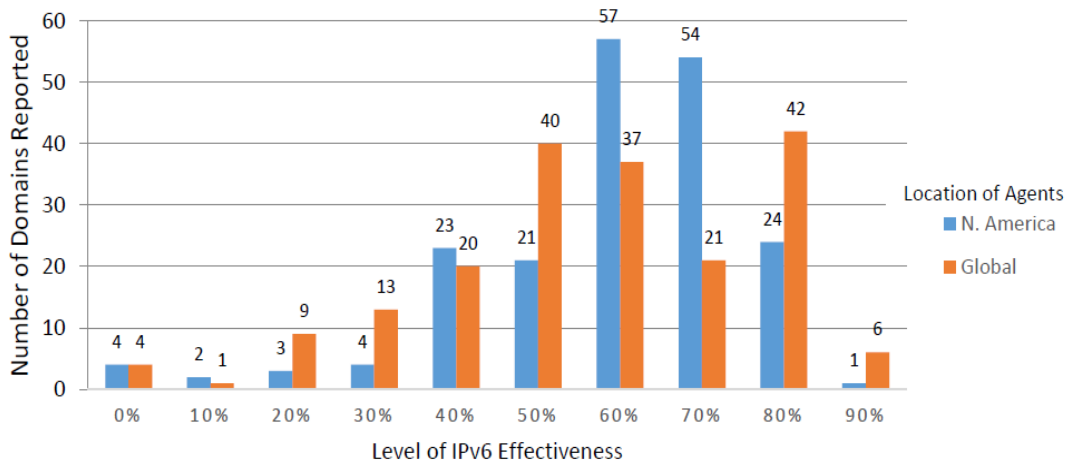

Figure 3. Average IPv6 effectiveness of USG agencies measured over six days [12]

The agents performed the following tasks/measurements every 10 minutes:

- DNS: request and reply

- IP: TCP connections to the web server

- HTTP: download time for all resources of the webpage.

The measurement results were recorded and the calculations were performed with the help of the V6Sonar tool, both regionally and globally (Figure 4).

\section{COMPARISON OF IPV6 HOSTING SERVICES}

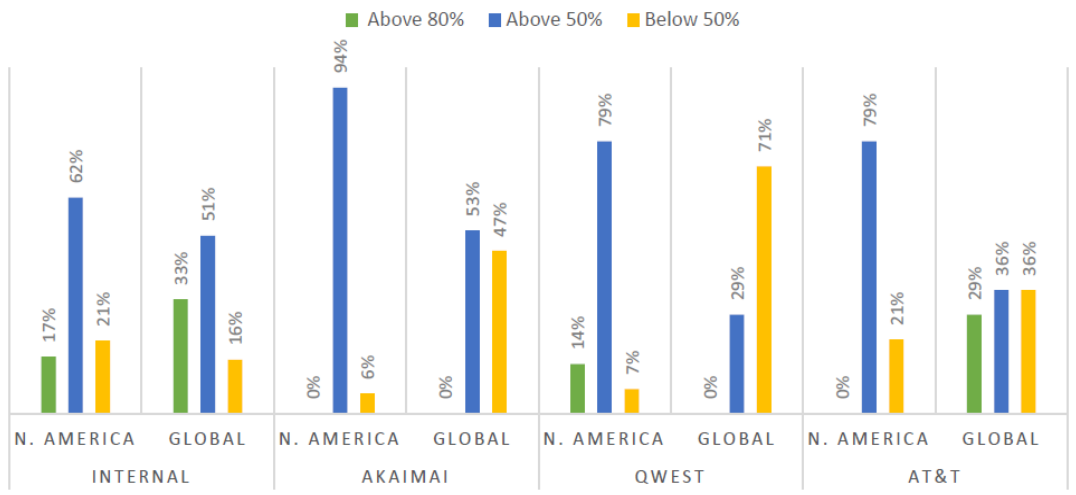

Figure 4. Comparison of IPv6 Hosting Services [12] 
Arthur Berger [14] examined the difference between IPv4 and IPv6 in terms of latency and packet loss. Berger carried out 44 million measurements between April and December 2010 using ping messages sent to nearly 7,000 globally distributed dual-stack name servers. The Table 3 summarizes the measurement results.

Table 3. Performance Comparison of v6 versus v4 [14]

\begin{tabular}{|c|c|c|c|c|c|}
\hline \multirow{2}{*}{$\begin{array}{l}\text { Geo- } \\
\text { Region }\end{array}$} & \multirow{2}{*}{$\begin{array}{l}\text { Set of } \\
\text { Nameservers } \\
\text { based on v6 } \\
\text { interface } \\
\end{array}$} & \multicolumn{2}{|c|}{ Mean Latency (ms) } & \multicolumn{2}{|c|}{ Mean Pocket Loss (\%) } \\
\hline & & $\mathrm{v} 4$ & v6 & $\mathrm{v} 4$ & v6 \\
\hline \multirow{3}{*}{$\begin{array}{l}\text { North } \\
\text { America }\end{array}$} & native & 52 & 95 & 0.4 & 2.1 \\
\hline & all & 55 & 101 & 0.6 & 3.2 \\
\hline & tunneled & 61 & 114 & 1.0 & 5.2 \\
\hline \multirow[t]{3}{*}{ Europe } & native & 154 & 163 & 0.5 & 0.7 \\
\hline & all & 158 & 168 & 0.7 & 1.8 \\
\hline & tunneled & 172 & 188 & 1.4 & 6.0 \\
\hline \multirow[t]{3}{*}{ Asia } & native & 205 & 212 & 3.0 & 1.2 \\
\hline & all & 216 & 240 & 2.8 & 2.7 \\
\hline & tunneled & 245 & 317 & 2.2 & 6.9 \\
\hline \multirow{3}{*}{$\begin{array}{l}\text { South } \\
\text { America }\end{array}$} & native & 188 & 208 & 0.8 & 1.0 \\
\hline & all & 186 & 235 & 1.7 & 4.4 \\
\hline & tunneled & 186 & 246 & 2.1 & 5.7 \\
\hline \multirow[t]{3}{*}{ Africa } & native & 337 & 350 & 2.0 & 4.8 \\
\hline & all & 356 & 379 & 2.8 & 6.7 \\
\hline & tunneled & 377 & 415 & 3.9 & 9.0 \\
\hline \multirow[t]{3}{*}{ Australia } & native & 211 & 232 & 0.4 & 1.0 \\
\hline & all & 216 & 244 & 0.6 & 2.0 \\
\hline & tunneled & 235 & 288 & 1.4 & 5.6 \\
\hline
\end{tabular}

The measured values describe an interesting situation: while in North America the delay of IPv6 is approximately twice of the delay of IPv4, in South America it is only $\sim 30 \%$ higher, but in the other regions the two values are only slightly different. The reason for the lower performance of the new generation protocol is most often due to the construction of the intermediate networks. Even though both endpoints of a connection can use IPv6 if there is a non-native IPv6 path between them. The use of transition techniques cause significant delays in daily life due to repackaging and address changes. This extra delay is also visible in the measurement results. The largest difference $(\sim 40 \%)$ between native and tunnel IPv6 access is in the Asian region. Aspect of packet loss rate, IPv4 also produced much better values. In the 
worst cases, IPv6 has five times higher packet loss rate compared to IPv4. Its root causes (similarly to those of delay) can be found in network architecture.

\section{Survey Methods}

In addition to the traditional information technology tools, some market research methods can be used, the most common of which are survey methods. A well-organized survey can be a cost-effective complementary or confirmatory tool in combination with other method(s). However, we do not recommend to rely on such data exclusively.

Pickard, Patrick and Robinson [15] selected 463 end-user business organizations in the Eastern region of North Carolina. To measure IPv6 readiness in those organizations, they sent a survey to the senior IT decision makers of the organizations. Data set is diverse and quite large in the geographical region, so this survey is considered representative in the given circumstances. The survey investigated the IPv6 readiness of each organization (detailed: Table 4) from several different perspectives. The data were processed and analyzed in accordance with the specifications and purpose: filter duplicate data, delete incomplete answers or answers from non-IT experts. According to final data, $23.5 \%$ of IT professionals have not even heard of IPv6 before the survey. Then other $76.5 \%$ were asked about a series of questions to recognize the stage of IPv6 readiness at their organization:

Table 4. IPv6 Readiness Stages [15]

\begin{tabular}{|l|l|}
\hline \multicolumn{1}{|c|}{ Stage } & \multicolumn{1}{c|}{ Criteria } \\
\hline 0. Not Aware & IT decision makers are not aware of IPv6. \\
\hline 1. Awareness & $\begin{array}{l}\text { IT decision makers are aware and have knowledge of } \\
\text { IPv6. }\end{array}$ \\
\hline 2. Interest & $\begin{array}{l}\text { IT decision makers are actively learning about IPv6 for } \\
\text { possible deployment within 12 months. }\end{array}$ \\
\hline 3. Evaluation/Trial & $\begin{array}{l}\text { The organization has initiated an evaluation or trial of } \\
\text { IPv6 in a test environment. }\end{array}$ \\
\hline 4. Commitment & $\begin{array}{l}\text { The organization has committed to adopt IPv6 through } \\
\text { establishment of a formal deployment plan. }\end{array}$ \\
\hline $\begin{array}{l}\text { 5. Limited } \\
\text { Deployment }\end{array}$ & $\begin{array}{l}\text { The organization has initiated an IPv6 project and has } \\
\text { completed deployment in at least one area of the } \\
\text { production environment }\end{array}$ \\
\hline $\begin{array}{l}\text { 6. General } \\
\text { Deployment }\end{array}$ & $\begin{array}{l}\text { The organization is using IPv6 in a substantial portion of } \\
\text { the production environment }\end{array}$ \\
\hline Rejecters & $\begin{array}{l}\text { Key IT decision makers are aware of IPv6, however the } \\
\text { organization has no plans to adopt at time of survey }\end{array}$ \\
\hline
\end{tabular}


$76.5 \%$ of respondents who had some level of knowledge of IPv6 rated the following level of readiness of their company based on the above six levels:

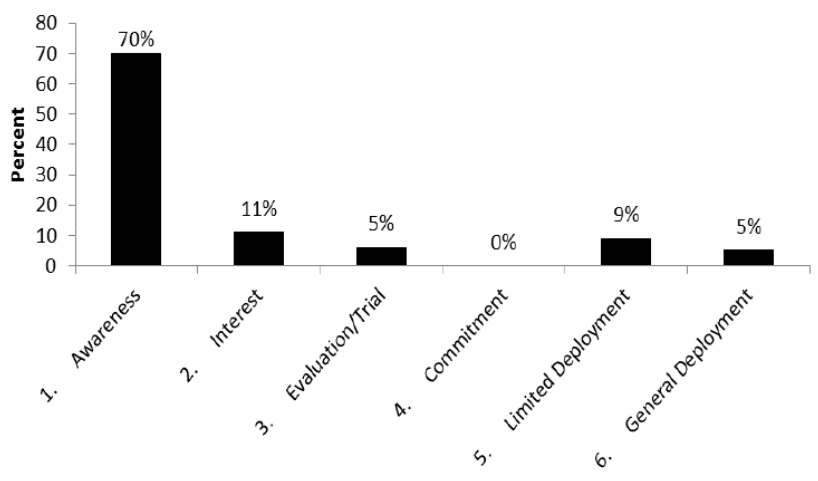

Figure 5. Highest achieved stage of organization IPv6 readiness [15]

The answers show that most organizations still do not feel the need for transition, and often are unaware of the opportunity. Specific numbers may not apply to other geographic regions, but unfortunately there is a similar level of general awareness and lack of development.

Another questionnaire survey was reported by Jordi Palet [16] in 2018. The questionnaire used for data collection is currently available from [17]. Respondents are employees or/and clients of ISPs. Most of the described networks in the responses are "own", that is, where the respondent is working or which services he/she is using. In addition, most networks have both IPv4 and IPv6 allocations. 105 countries participated in this survey. Networks have different technical maturity, so IPv6 deployment is mostly between trial and long-term commercial usage. According to the results, $62 \%$ of allocated Wide Area Network (WAN) prefixes are 64 bit long (/64), but some of the addresses are allocated for later usage. Based on the number of allocated WAN prefixes, the proportion of IP versions in the examined dataset: IPv4 - 1076 (69\%) and IPv6 - 483 (31\%).

The following data collection was not a conventional survey. In this case, instead of a questionnaire, a shared Excel spreadsheet was used, that is available in the IETF v6ops mailing list [18][19]. Filling in is voluntary and most contributors indicate the source, see Table 5. 
Table 5. Summary of the Sources of the Self-Declaration Survey Data Sources Published in IETF v6ops Mailing List [19]

\begin{tabular}{|l|c|}
\hline \multicolumn{1}{|c|}{ Dataset } & $\begin{array}{c}\text { Number of occurrences in the } \\
\text { dataset }\end{array}$ \\
\hline Facebook discussion groups & 27 \\
\hline Internet sources (e.g. websites) & 19 \\
\hline Different persons (in writing, face-to-face) & 7 \\
\hline Different sources & 2 \\
\hline RIR network information & 3 \\
\hline User test, own router & 2 \\
\hline Company information & 13 \\
\hline Source not listed & 9 \\
\hline
\end{tabular}

Table 6 gives some information about the deployment of different transition technologies and methods. The number of fillers is relatively small (82 entries in the table at the time of writing) and the information mainly concerns participants in the IT sector, therefore, this survey is not representative - but rather gives an approximate picture of the prevalence of transition methods and current trends.

Table 6. Summary of Transition Mechanisms for Self-Reporting Survey Published on IETF v6ops Mailing List [19]

\begin{tabular}{|l|c|}
\hline \multicolumn{1}{|c|}{ Transition Mechanism } & Number of occurrences in the dataset \\
\hline Dual Stack & 35 \\
\hline Dual Stack + MAP-T & 3 \\
\hline Dual Stack + Dual Stack Lite & 3 \\
\hline Dual Stack + NAT64 & 2 \\
\hline Dual Stack + lw4o6 & 1 \\
\hline Dual Stack Lite & 18 \\
\hline 6rd & 6 \\
\hline 464XLAT & 8 \\
\hline 464XLAT + NAT64 & 3 \\
\hline MAP-E & 1 \\
\hline lw4o6 & 1 \\
\hline IPv4-only & 1 \\
\hline
\end{tabular}

As Table 6 shows, IPv4-only access is increasingly rare among larger telecommunications companies. The time to complete deployment is unknown, so service providers will do their best to serve their clients, who are still using IPv4 as well as those, who are already using IPv6 capable devices. There is a wide range of 
IPv6 transition technologies, although, with the continued spread of IPv6, some methods are sometimes more popular and then get out of use.

\section{Complex Measurement Methods}

Eravuchira et al. [20] made measurements on the Alexa's top 100 dual stack web pages using webget and simweb tools. The study focused on the complexity of content and service.

The main properties observed by the webget are DNS lookup time, the time until the first byte appears, HTTP request duration, content size and download speed.

Simweb also monitored the size of the content, the content type, source URL, IP endpoint, CURL response and HTTP status codes. The simweb test was run over IPv4 and IPv6, repeated every hour. The data set used for the analysis comes from the results of 65 days of simweb surveys collected between April 2015 and June 2015.

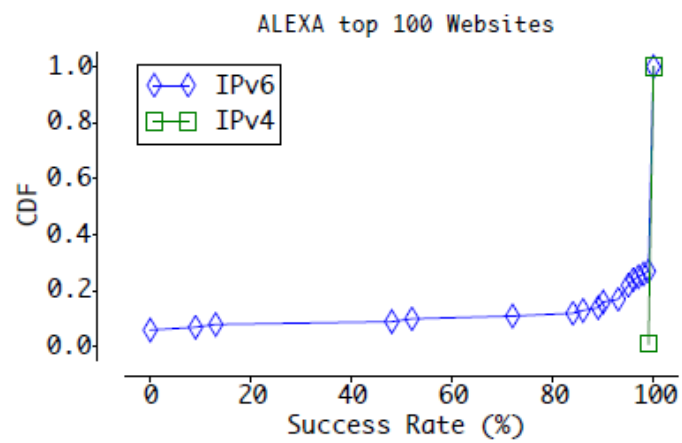

Figure 6. Distribution of success rates towards ALEXA top 100 dual-stacked websites [20]

As Figure 6 shows, for ALEXA top 100 dual stack measurements over IPv4, the median success rate was $100 \%$ over the entire measurement period, except for one web page. Above IPv6, 27\% of webpages have errors, 9\% have a 50\% error rate, and $6 \%$ have a $100 \%$ error rate.

Between 2004 and 2014, Czyz et al. [21] examined the transition process from the perspective of different players on the Internet: content provider, ISP and content consumer. The measurement indicators considered are summarized in the Table 7. 
Table 7. Indicators from the Perspective of the Main Stakeholders of the Internet [21]

\begin{tabular}{|l|l|}
\hline \multicolumn{1}{|c|}{ Perspective } & Prerequisite IP Functions and Operational Characteristics \\
\hline $\begin{array}{l}\text { Content } \\
\text { Provider }\end{array}$ & $\begin{array}{l}\text { Nameservers } \\
\text { Transition Technologies } \\
\text { Server Side Readiness }\end{array}$ \\
\hline & Transition Technologies \\
& Address Allocation \\
Service & Address Advertisement \\
Provider & Resolvers \\
& Traffic Volume \\
& Network RTT \\
& Topology \\
\hline Content & Application Mix \\
Consumer & Queries \\
\hline
\end{tabular}

Figure 7 and Figure 8 show the measurement results for two selected metrics. Additional data for the test [21].

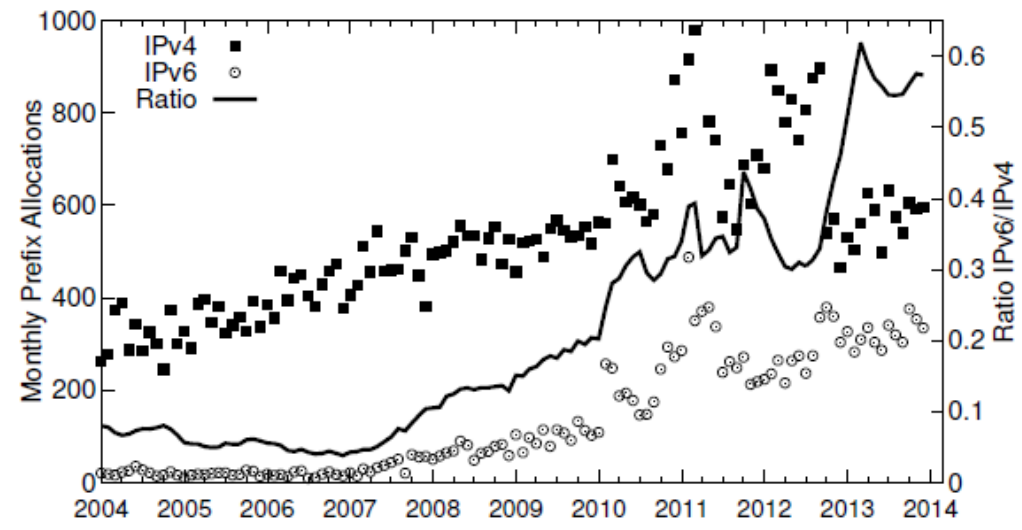

Figure 7. Number of allocated prefixes [21]

Figure 7 shows the change in the number of allocated but not necessarily used prefixes. In 2004, at the beginning of the measurement cycle, approximately 300 IPv4 prefix allocations per month occurred. Then in 2011, the value jumped to 8001000 per month, thereafter dropped to around 500 by 2013 . In order to put these 
numbers into context, it is worth mentioning that the data set used initially contained 69,000 and 10 years later 136,000 prefix allocations. So in the observed 10 years, the number of IPv4 prefix allocations has almost doubled. On the contrary, the number of monthly IPv6 prefix allocations until about the first quarter of 2007 was rather low and varying, but growth continues to increase thereafter. Overall, the share of monthly allocated IPv6 prefixes has been rising since late 2013, while the proportion of IPv4 has declined.

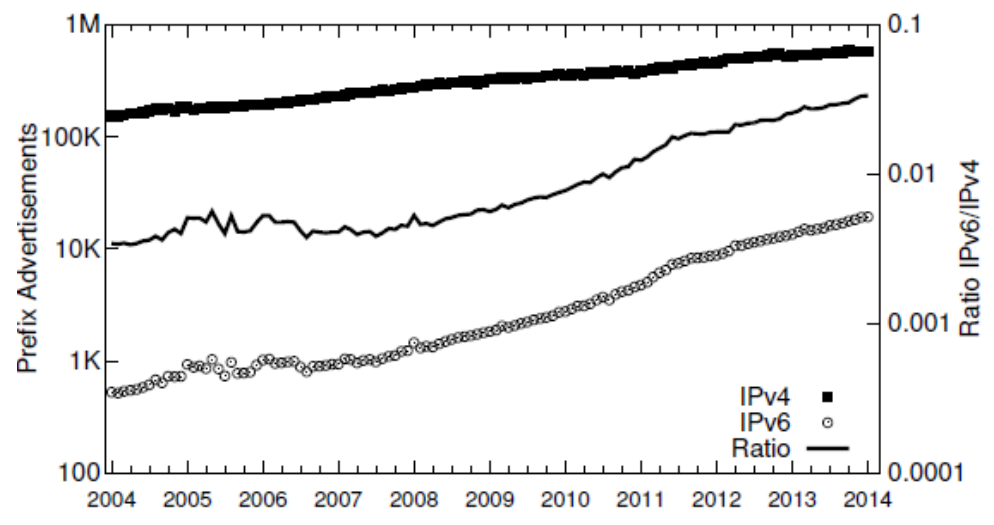

Figure 8. Number of advertised prefixes [21]

Figure 8 shows the change in the number of advertised prefixes. On January 1, 2004 , there were only 526 advertised IPv6 prefixes, compared to 19,278 on January 1, 2014. However, 153,000 IPv4 prefixes were announced in early 2004 and 578,000 in early 2014. It is noticeable that although specific numerical values for IPv4 are higher, the rate of growth of IPv6 is increasing.

Pickard and Southworth [22] aimed to make their research as similar as possible to that of Czyz et al. [21]. To do this, 8 of the metrics studied by Czyz et al. were selected and could continue to be monitored. We call it a continuation because Czyz and co-workers completed their measurements in January 2014, when Pickard and Southworth started their work. So for the 8 properties selected, data collection was ongoing until December 2015. The Table 8 shows the metrics selected by the Pickards, the source of the data, and the time of collection. It is important to mention: IX traffic is does not provide correct measurement results, because IX is mainly change the ISPs' traffic. 
Table 8. Summary of datasets related metrics and time period of measurement [22]

\begin{tabular}{|l|l|c|}
\hline \multicolumn{1}{|c|}{ Dataset } & \multicolumn{1}{c|}{ Metric } & Time Period \\
\hline RIR Prefix Allocations & Address Allocation & Jan 2014 - Dec 2015 \\
\hline Route Views AS6447 & Network Advertisements & Jan 2014-Dec 2015 \\
\hline Hurricane Electric & Authoritative Nameservers & Dec 2015 Snapshot \\
\hline Route Views AS6447 & Topology & Jan 2014- Dec 2015 \\
\hline Alexa Top Hosts & Server Side Readiness & Dec 2015 Snapshot \\
\hline Google IPv6 Stats & Client Side Readiness & Jan 2014- Dec 2015 \\
\hline $\begin{array}{l}\text { Amsterdam Internet } \\
\text { Exchange (AMS-IX) }\end{array}$ & Traffic Volume & Jan 2014 - Dec 2015 \\
\hline Alexa Top 500 Hosts & Performance & Dec 2015 \\
\hline
\end{tabular}

Figure 9 and Figure 10 show the measurement results for two selected metrics. Additional data for the test [22].

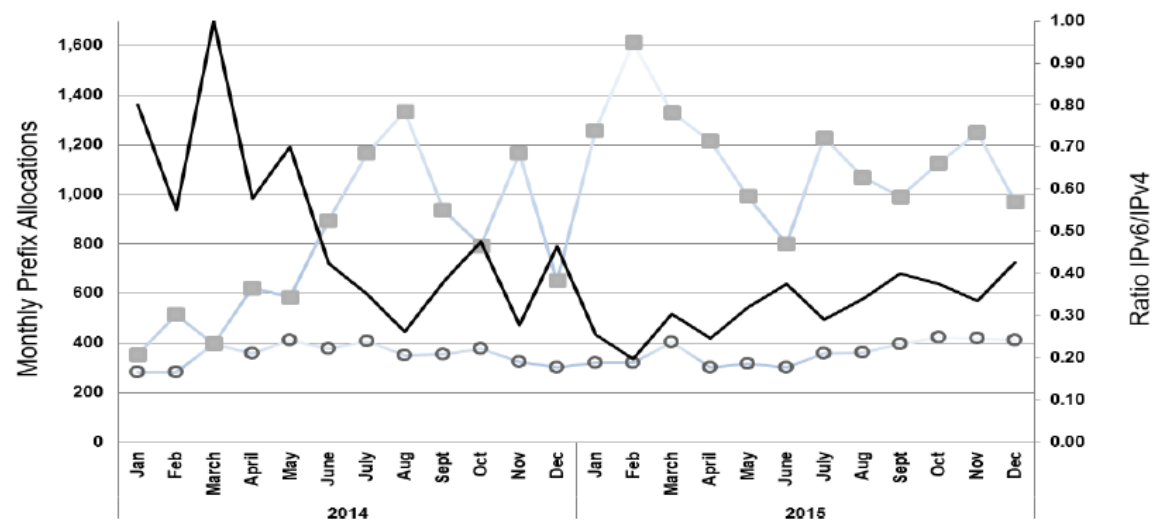

O-IPv6 Allocations

IPv4 Allocations

-Ratio:

Figure 9. Number of prefix allocations by month aggregated from all RIRs. [22]

Figure 9 shows the aggregate number of RIRs' monthly prefix allocations between January 2014 and December 2015. As can be seen, the number of IPv6 allocations increased from 300 per month to 400 per month in two years, with relatively low intermediate fluctuations. In contrast, there are several significant fluctuations in the number of IPv4 allocations. Larger peaks coincide with periods when IANA returned IPv4 address blocks to RIRs. The significant decrease in the IPv6 / IPv4 ratio is also due to the release of more (re) redistributable IPv4 prefixes, while allocating IPv6 prefixes at a relatively stable rate. 


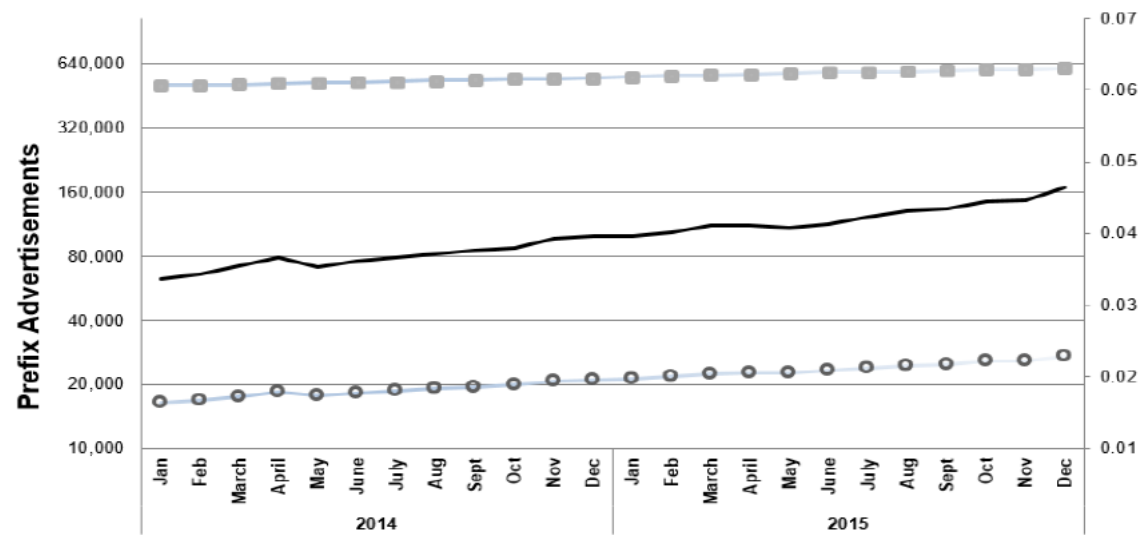

O-IPv6 Allocations

-IPv4 Allocations -Ratio:

Figure 10. Prefixes seen in the Global BGP table of Route Views AS 6447[22]

Figure 10 shows the change of the number of IPv4 and IPv6 prefixes advertised by a global BGP routing table and their proportion. The number of advertised IPv6 prefixes was 16,537 in January 2014, and 27,236 in December 2015, an increase of $65 \%$ over two years. At the same time, the number of IPv4 prefix advertisements increased from 491,000 to 580,000 , an increase of $18 \%$. Proportion of number of advertised IPv6 and IPv4 prefixes increased from 0.033 to 0.046 , which was more strongly influenced by the growth rate of IPv6 prefix advertising.

Table 9. Measure of operational characteristics of each metric beginning January 2014 and ending December 2015 [22]

\begin{tabular}{|l|c|c|c|}
\hline \multicolumn{1}{|c|}{ Metric/Measured Aspect } & \multicolumn{2}{c|}{ IPv6 Status } & \\
\hline & Jan 2014 & Dec 2015 & Change \\
\hline IPv6 Prefix Allocation & 18,180 & 26,481 & $+46 \%$ \\
\hline IPv6 to IPv4 Prefix Allocation Ratio & 0.13 & 0.17 & $+31 \%$ \\
\hline Announced IPv6 Prefixes & 16,537 & 27,236 & $+65 \%$ \\
\hline IPv6 to IPv4 Announced Prefix Ratio & 0.033 & 0.046 & +39 \\
\hline Authoritative Nameservers & $91 \%$ & $97,6 \%$ & $+7 \%$ \\
\hline Topology & 7,905 & 10,862 & $+37.4 \%$ \\
\hline Unique IPv6 AS Paths & 122,160 & 305,100 & $+150 \%$ \\
\hline Server Side Readiness & 320 & 830 & $+88 \%$ \\
\hline Client Side Readiness & $2.5 \%$ & $9 \%$ & $+260 \%$ \\
\hline IPv6 Traffic Volume & $15 \mathrm{~Gb} / \mathrm{s}$ & $35 \mathrm{~Gb} / \mathrm{s}$ & $+133 \%$ \\
\hline
\end{tabular}


Like Czyz and co-workers, Pickard and Southword found that the proportion of IPv6 is steadily increasing at an accelerated rate for each tested metric.

\section{Summary}

As we have seen, some of the methods, metrics and tools presented have been used by several researchers (groups) to investigate the penetration of next-generation Internet Protocol. In addition, there are several tools that are quite different in each study. This many to many connection is made more transparent by Table 10 .

Table 10. Summary of Measurement Methods, Tools and Metrics in Paper

\begin{tabular}{|l|l|}
\hline \multicolumn{1}{|c|}{ Mechanism/metric/tool } & \multicolumn{1}{|c|}{$\begin{array}{c}\text { Reference in } \\
\text { this document }\end{array}$} \\
\hline Applications (pl. V6Sonar, Simweb, Webget) & {$[12][21][20]$} \\
\hline Transition Mechanisms & {$[21][18]$} \\
\hline Autonomous Systems (prefixes, paths) & {$[5]$} \\
\hline Address allocations and announces & {$[1][21][22]$} \\
\hline $\begin{array}{l}\text { Resource Record values of DNS queries and replies, latency } \\
\text { of DNS resolution }\end{array}$ & {$[5][8][12][21]$} \\
\hline Traffic of DNS resolvers and name servers & {$[11][21][22]$} \\
\hline Traffic volume & {$[21][22]$} \\
\hline Network announcements (BGP routing tables) & {$[22]$} \\
\hline Survey & {$[15][16]$} \\
\hline Client side readiness, number of clients & {$[8][21][22]$} \\
\hline Server side readiness & {$[21][22]$} \\
\hline Quality of Service (e.g. RTT, HTTP load time, response time) & {$[5][14][20][21]$} \\
\hline
\end{tabular}

As we have seen with complex measurement methods, it may sometimes be worth combining different technologies and tools. Not only can we collect more data, but different types of information greatly contribute to the correct interpretation of the data and allow us to draw valuable conclusions. Overall, therefore, it is not possible to unambiguously identify a universally best technique, and each method has its own field of application. The choice of the appropriate method(s) depends largely on the data and tools available to us. Table 11 shows the metrics already described in the 
previous sections, grouped by data availability and the length of time needed to detect change.

Table 11. Classification of Metrics by Scope and Measurement Period

\begin{tabular}{|l|c|c|}
\hline \multicolumn{1}{|c|}{ Mechanism/metric/tool } & $\begin{array}{c}\text { Access to the } \\
\text { Dataset }\end{array}$ & Period of Time \\
\hline DNS messages (e.g. RR, latency) & public & months \\
\hline $\begin{array}{l}\text { Performance (e.g. network RTT, latency, } \\
\text { pocket loss) }\end{array}$ & public & months \\
\hline Number of prefix allocations & public/private & years \\
\hline Number of prefix announcements & public/private & years \\
\hline Applications & public & months \\
\hline Alexa Rankings & public/limited & years \\
\hline Survey questionnaire & limited & months/years \\
\hline Prefix allocations & private & years \\
\hline Prefix announcements & private & years \\
\hline Traffic volume & private & months \\
\hline Topology & private & months \\
\hline Nameservers, resolvers & private & months \\
\hline AS paths & private & months \\
\hline Transition technologies & private & years \\
\hline
\end{tabular}

There is public access to a data set, when it is freely available to anyone. We consider limited access to different surveys and non (completely) free datasets. Private data is typically owned by service providers and is generally not freely accessible, though there are exceptions [23].

\section{Conclusion}

We have surveyed the methods available for measuring the deployment of IPv6. We have classified them from different aspects. According to the type of the measurements, we have distinguished client side, server side, survey and complex methods. Of course, different types of methods work from different sets of data that 
are not accessible in the same way. The measurement methods presented can also be classified according to the availability of the data source used: we distinguished public, restricted, and private sources of data. Another important feature for choosing the right measurement method is the time interval required for collecting the amount and quality of data to be evaluated. Although a proper analysis of most of the data provides a picture of the current situation, monitoring and data collection may take up to several months, in some cases up to several years, to draw conclusions and prepare forecasts about the trends. We know that our list is not exhaustive and there are countless other classification options. With our three different viewpoints, we wanted to offer more options and help researchers to choose the most appropriate tool(s). We hope that our work can be a good starting point for further investigations.

\section{Acknowledgement}

The results presented in this paper arose from the BSc final project thesis of the first author under the supervision of the second author.

\section{References}

[1] NRO. Free Pool of IPv4 Address Space Depleted. (2019)

URL https://www.nro.net/news/ipv4-free-pool-depleted

[2] IPv4.Global.

URL https://auctions.ipv4.global

[3] S. Deering, R. Hinden, Internet Protocol, Version 6 (IPv6) Specification, IETF RFC 2460 (1998)

doi: https://doi.org//10.17487/RFC2460

[4] Google IPv6 Statictics.

URL https://www.google.com/intl/en/ipv6/statistics.html

[5] M. Gregr, M. Sveda, T. Podermanski, Measuring Quality and Penetration of IPv6 Services, in: ICNS 2014: The Tenth International Conference on Networking and Services (2014).

URL https://www.researchgate.net/publication/261983397_ Measuring_Quality_and_Penetration_of_IPv6_Services

[6] E. Karpilovsky, A. Gerber, D. Pei, J. Rexford, A. Shaikh, Quantifying the Extent of IPv6 Deployment, in: S.B. Moon, R. Teixeira, S. Uhlig (Eds.), 
Passive and Active Network Measurement. Lecture Notes in Computer Science, vol 5448. Springer Berlin, Heidelberg, 2009.

doi: https://doi.org//10.1007/978-3-642-00975-4_2

[7] Alexa Top 500 Global Sites

URL https://www.alexa.com/topsites

[8] Alexa top 1 million sites directly from the server

URL https://gist.github.com/chilts/7229605

[9] G. Huston, Testing IPv6 for World IPv6 Day (2011).

URL http://www.potaroo.net/ispcol/2011-05/ip6test.htmI

[10] Google Analytics

URL https://analytics.google.com

[11] G. Huston, Counting IPv6 in the DNS (2012)

URL https://labs.apnic.net/?p=266

[12] J. Pickard, D. Stocks, R. Hamman, A. Robinson, Study on the Quality of IPv6 Enablement of US Government Websites, 2015

URL http://www. nephos6.com/pdf/Sonar-Technical-Report-USGov.-IPv6-Websites-Ver.-3.1-dp.pdf

[13] Nephos6

URL https://www.nephos6.com/

[14] A. Berger, Comparison of Performance over IPv6 vs. IPv4, Akamai Technologies (2011)

URL http://ftp.caida.org/workshops/isma/1202/slides/ aims1202_acox_supplement.pdf

[15] J. Pickard, A.Y. Patrick, A. Robinson, Analysis of Enerprise IPv6 Readiness, in: Proceedings of the IEEE SoutheastCon, Fort Lauderdale, Florida (2015) doi: https://doi.org//10.1109/SECON.2015.7133016

[16] J. Palet Martinez, IPv6 Deployment Survey (Residential/Household Services). How IPv6 is being deployed?, APNIC 44 (2017)

URL https://indico.uknof.org.uk/event/41/contributions/ 542/attachments/712/866/bcop-ipv6-prefix-v9.pdf 
[17] J. Palet Martinez, IPv6 Deployment Survey (Residential/Household Services). How IPv6 is being deployed? (2017)

URL http://survey.consulintel.es/index.php/175122

[18] L. Howard, [v6ops] discussion of transition technologies, IETF v6ops mailing list (2018)

URL https://www.ietf.org/mail-archive/web/v6ops/current/ msg28889.htmI

[19] L. Howard, "[v6ops] Transition mechanisms in use", IETF v6ops mailing list (2018)

URL https://www.ietf.org/mail-archive/web/v6ops/current/ msg29096.html

[20] S.J. Eravuchira, V. Bajpai, J. Schönwälder, S. Crawford, Measuring Web Similarity from Dual-stacked Hosts, in: CNSM Mini-Conference Paper, 2016 doi: https://doi.org//10.1109/CNSM.2016.7818415

[21] J. Czyz, M. Allman, J. Zhang, S. Iekel-Johnson, E. Osterweil, M. Bailey, Measuring IPv6 Adoption, ACM SIGCOMM Computer Communication Review, 2014.

doi: https://doi.org//10.1145/2619239.2626295

[22] J. Pickard, J.B. Southworth, The State of IPv6: Measuring Global Adoption, in: ASEE's 123rd Annual, Conference \& Exposition, New Orleans, LA, 2016 doi: https://doi.org//10.18260/p.27022

[23] APNIC Labs Resource Reports Archive.

URL https://labs.apnic.net/dists/archive/ 